\title{
Prognosis and natural history of Reiter's syndrome
}

\author{
ANDREI CALIN, ROBERT FOX, ROBERT C. GERBER, \\ AND DAVID J. GIBSON
}

From the Stanford University Medical Centre, California, USA

Statements such as 'joint symptoms are characteristically acute, short-lived, and transient' ${ }^{330}$ may give the patient and physician a false expectation. For the occasional, fortunate individual Reiter's syndrome (RS) may be a self-limiting disease. In fact, the prognosis of RS is notoriously difficult to assess. The reasons for this are protean. (1) Many patients are young men who form a mobile population and long-term follow-up is difficult. (2) In many patients, especially women, the disorder is misdiagnosed as 'seronegative rheumatoid arthritis' rather than RS. (3) Often the early classical stigmata of RS are forgotten and one is left with a patient complaining of a persistent arthropathy and perhaps spinal disease. The diagnosis of 'ankylosing spondylitis' is used. (4) Medical care is fragmentary; patients may be followed up by the orthopaedic surgeon, ophthalmologist, or other specialists. Long-term follow-up studies are therefore few and suffer from the inherent problem that they are usually performed in academic centres where patients with more severe disease are followed up.

\section{Review of studies}

A review of reported studies suggests that the prognosis of RS is relatively poor. The original Paronen epidemic ${ }^{244}$ in 1944, which affected 344 patients, was reviewed 20 years later, ${ }^{267}$ when 100 patients were available for study (no doubt including a bias towards those with more severe disease). Eighty of these patients still had active disease, which included 32 with ankylosing spondylitis, 18 with recurrent acute arthropathy, 30 with chronic joint disease, 7 with recurrent uveitis, and 2 with aortic regurgitation. Some 40 (over $10 \%$ of the original RS patients) were unable to work because of persistent RS.

Csonka (see preceding paper, p. 24) followed up 185 patients for a period of 2 to 15 years. Sixty-two per cent had active disease, and he suggested a recurrence rate of $15 \%$ per patient per year. Good ${ }^{133}$ followed up 34 patients for 2 years: 24 had persistent disease at the end of the period, including 13 with sacroiliitis. Our follow-up ${ }^{55}$ of Noer's original study ${ }^{238}$ revealed that four of the five patients contacted had active and severe disease 13 years after the original epidemic. All four had persistent joint problems, two had deteriorating vision after iridocyclitis, and two had ankylosing spondylitis. Only one remained free from active disease. Of interest, this patient was the only one of the five who was HLA-B27 negative. McClusky et al $^{217}$ have also suggested that patients with HLA-B27 are more likely to develop severe disease.

\section{Our study}

Our more recent study, ${ }^{53}$ however, throws some doubt on this concept. In this investigation 113 patients were consecutively admitted to the study at both a university and a community medical centre in order to avoid selection bias (see Table 1 for diagnostic criteria). At a mean of 6.4 years 104 patients $(93 \%)$ were available for follow-up. In summary, there were no major differences between the two centres. There was little difference between men and women with RS or between B27-positive and negative patients (in contrast to our expectations).

The functional status at follow-up is shown in Table 2. Features at entry to the study were compared with these at follow-up examination (Figure). Polyarthritis persisted in $83 \%$ of cases, urethritis or cervicitis, or both, in $42 \%$, back pain and heel pain in about $50 \%$, eye disease in $33 \%$, balanitis and mouth ulcers in $25 \%$, and other manifestations as

\section{Table 1 Diagnostic criteria for Reiter's syndrome}

(1) Seronegative asymmetric arthopathy (predominantly lower
extremity) plus (2)
(2) One or more of the following:
Urethritis/cervicitis
Dysentery
Inflammatory eye disease
Mucocutaneous disease (balanitis or oral ulceration or
keratodermia)
(3) Exclusions
Primary ankylosing spondylitis
Psoriatic arthropathy
Other rheumatic disease


Table 2 Follow-up data on 104 Reiter's syndrome patients at mean of 6.4 years

\begin{tabular}{lll}
\hline & $n=104$ & $\%$ \\
\hline No symptoms & 17 & 16 \\
Annoying symptoms & 23 & 22 \\
Interference with job & 36 & 35 \\
Forced to change job & 16 & 15 \\
Unemployable & 12 & 12 \\
\hline
\end{tabular}

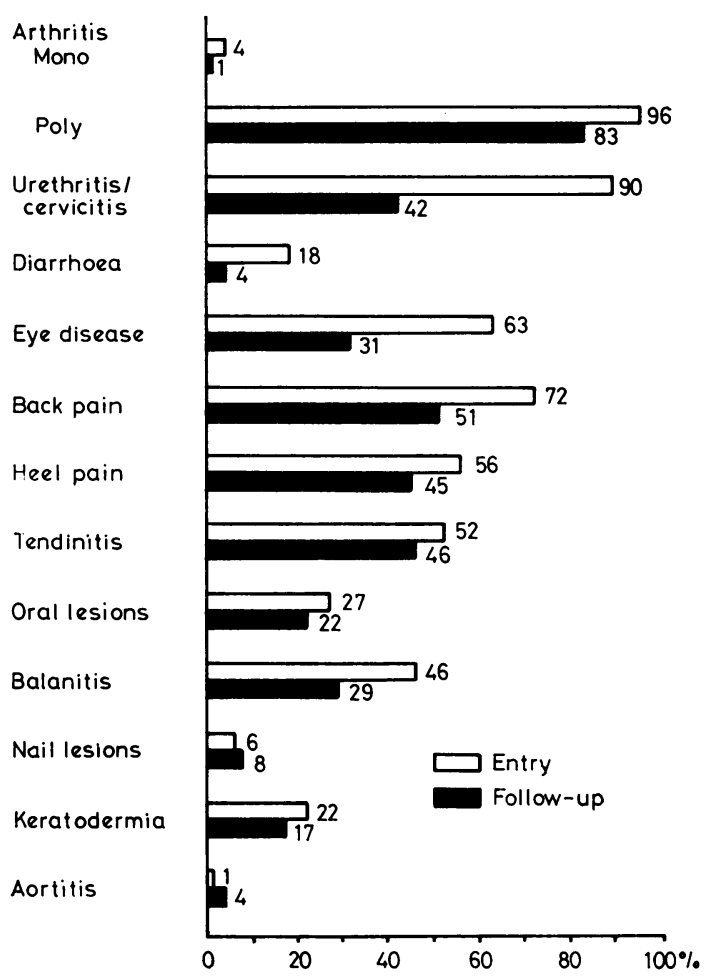

Figure Evaluation of 104 patients at follow-up at mean of 6.4 years. Numbers at end of bars refer to percentage of total with manifestation.

shown. Some patients have recurrent episodes with asymptomatic periods between exacerbations. However, in our experience, most have continuing but sometimes minimal background discomfort. These symptoms may include back pain, heel pain, or other problems. Many of our patients had a continuing chronic disease from the outset. Some recurrences were precipitated by sexual indescretion, but many appeared after a diarrhoeal episode or de novo with urethritis appearing as an 'allergic' phenomenon rather than an infective event.
To try to define prognostic variables at onset we compared features in patients who developed lasting disabilities with features in those who had self-limiting disease. Ankle and heel pain were the major differences. Both were more common in those with a poor prognosis. ${ }^{53}$ Thus RS is a major chronic rheumatic disease. The physician and patient must have a realistic concept of its natural history.

\section{Conclusion}

Future works of reference should not include such statements as 'The disease is usually self-limited, subsiding in six weeks to six months and complete remission with full recovery of peripheral joint function is the rule'. ${ }^{249}$

\section{General discussion}

DR. T. L. VISCHER: How many women are there in your study? Did women with B27 behave differently from men and did you have any women homozygus for B27?

DR. CALIN: We have data on only 13 women, not enough to compare those with and without B27.

PROF. A. S. RUSSELL: In the eventual outcome you? mention that $83 \%$ of your patients have synovitis or even polyarticular disease. Did all of these $83 \%$ have actual synovitis at the time you saw them, or did that include synovitis by history?

DR. CALIN: 'Synovitis' refers to objective findings at the time of follow-up examination. This may have been minimal such as, for example, a sausage digit.

PROF. RUSSELL: So there must have been others who had recurrent episodes which were entirely silent when you saw them.

DR. CALIN: Yes.

DR. E. ALBERT: Was there a typical initial pattern of symptoms at the beginning of the disease which would be kept up to the end of the study or would characteristically reappear during relapses? In other words, if somebody first presented with eye disease did he tend to have eye disease at the end of the study as well?

DR. CALIN: That is an important point, although we have not systematically investigated it as yet. The initial disease pattern did not always persist. Some people had relatively little eye disease at onset and presented with major eye problems two to three years later. Where the pattern did continue was in the rheumatic features such as back pain, ankle arthritis, and plantar fasciitis, which turned out to be the major chronic problems. 
PROF. M. ZIFF: You have tabulated a list of findings at onset of the disease and at the end but one doesn't get the picture of whether RS is, in your experience, a remitting disease, whether the patients at the end are better off than they were in their initial attacks, or are they chronic all the way?

DR. CALIN: It seems that there is often a continuous background chronicity with occasional exacerbations. Patients with plantar fasciitis, heel pain, and back pain have more chronic symptoms and a worse prognosis. Of course, some fortunate patients do have a self-limiting disease.

PROF. ZIFF: But when you tabulate $80 \%$ or $90 \%$ polyarthritis at the end of the period of observation one gets the impression that the patients have polyarthritis over a long period of time.

DR. CALIN: Yes, but the polyarthritis component may be minimal and cause little loss of function. 